\title{
Influencia de la consejería nutricional en el colesterol total de trabajadores de la Clínica San Juan de Dios, Lima 2017
}

\section{Influence of the nutritional counseling on total cholesterol levels of workers from Clinica San Juan de Dios, Lima 2017}

\author{
Milagro Bellido Vallejo ${ }^{1, a}$, Juan Alfredo Palaco Vásquez ${ }^{2,3, b}$, Luzmila Troncoso-Corzo,c \\ Universidad Nacional Mayor de San Marcos. Lima, Perú. \\ 2 Universidad Tecnológica del Perú. Lima, Perú. \\ ${ }^{3}$ Universidad Católica de Santa María. Arequipa, Perú. \\ a Magister en Nutrición Clínica, ORCID: https://orcid.org/0000-0002-9578-8595 \\ ${ }^{b}$ Magíster en Gestión del Desarrollo y Gerencia Social, ORCID: https://orcid.org/0000-0002-3163-2030 \\ c Doctora en Medicina, ORCID: https://orcid.org/0000-0003-1075-874X
}

\section{An Fac med. 2020;81(1):131-2. / https://doi.org/10.15381/anales.v81i1.17257}

\section{Correspondencia: \\ Milagro Bellido Vallejo \\ kajolbv@hotmail.com}

Recibido: 8 de noviembre 2019 Aprobado: 30 de diciembre 2019 Publicación en línea:31 de marzo 2020

Conflictos de interés: Los autores declaran no tener conflictos de interés

Fuentes de financiamiento:

Autofinanciado

Citar como: Bellido M, Palacio J, Troncoso-Corzo L. Influencia de la consejería nutricional en el colesterol total de trabajadores de la Clínica San Juan de Dios, Lima 2017. An Fac med. 2020;81(1):131-2. DOI: https://doi. org/10.15381/anales.v81i1.17257

\section{Sr. Editor,}

La consejería nutricional evidencia un proceso comunicacional, interpersonal y participativo entre el nutricionista y el paciente. Estudios previos demuestran que esta intervención tiene efecto en el cambio de dieta, niveles de lípidos y presión arterial ${ }^{(1)}$. Así, la nutrición pasó de brindar intervenciones desde un enfoque preventivo, a una perspectiva terapéutica en el caso de patologías crónicas, lo que facilita tomar decisiones nutricionales que mejoren el control glicémico, presión arterial, colesterol total (CT) y el índice de masa corporal (IMC) ${ }^{(2)}$.

Presentamos los resultados de una investigación que tuvo como objetivo determinar la influencia de la consejería nutricional en el colesterol total de trabajadores de la Clínica San Juan de Dios, con un universo de 87 trabajadores en diferentes áreas. El plan de consejería se realizó en tres etapas diseñadas para orientar al colaborador y familiares directos. Durante la sesión de consejería se priorizó el uso de la dieta mediterránea (Dmed) la cual ha mostrado solvencia científica, por asociación o intervención, siendo considerado como un patrón dietario saludable ${ }^{(3,4)}$.

El estudio obtuvo una media de IMC antes de la consejería igual a $27 \mathrm{~kg} / \mathrm{m}^{2}$, la que disminuyó a $26,2 \mathrm{~kg} / \mathrm{m}^{2}$, después de la intervención. La mayoría inicio con sobrepeso y obesidad I (45\% y $18 \%$ respectivamente); posterior a la consejería, los diagnósticos de obesidad tipo I bajaron en-8\% y se observó variación al estado nutricional normal (+10\%).

Los valores iniciales altos y muy altos de colesterol estuvieron presentes en $74 \%$ de trabajadores; mientras que, al finalizar la intervención estos valores disminuyeron un-13\%. Al comparar las características de la población objetivo con los valores de IMC y colesterol total, se evidenció que, la consejería nutricional disminuyo en promedio 1,9 kg de peso y $12 \mathrm{mg} / \mathrm{dL}$ de CT.

Los varones jóvenes (edad <39 años) que laboraban como personal de atención médica, evidenciaron mayores cambios en el peso final; asimismo, la disminución de colesterol fue mayor en mujeres adultas y adultas mayores que laboraban como administrativos y personal de apoyo (Tabla 1). Se observó que todos los indicadores medidos exponen relación estadísticamente significativa respecto al tratamiento $(p<0,05)$.

La mayoría de los trabajadores estuvieron con sobrepeso y obesidad; este grupo de trabajadores tuvieron CT de nivel alto (33\%) y muy alto (14\%). Asimismo, se encontró que a medida que evoluciona el IMC aumentan los valores de CT, tendencia similar a la evidenciada 
Tabla 1. Variación entre los promedios del IMC, peso, talla y colesterol total después de programa de consejería nutricional a trabajadores de la Clínica San Juan de Dios, Lima 2017.

\begin{tabular}{|c|c|c|c|c|c|c|c|}
\hline \multirow{2}{*}{ Características } & IMC Inicial & IMC Final & Peso Inicial & Peso Final & Talla & $\begin{array}{l}\text { Colesterol } \\
\text { Inicial }\end{array}$ & $\begin{array}{l}\text { Colesterol } \\
\text { Final }\end{array}$ \\
\hline & $\mathrm{Kg} / \mathrm{m}^{2}$ & $\mathrm{Kg} / \mathrm{m}^{2}$ & $\mathrm{Kg} / \mathrm{cm}$ & $\mathrm{Kg} / \mathrm{cm}$ & $\mathrm{cm}$ & $\mathrm{mg} / \mathrm{dL}$ & $\mathrm{mg} / \mathrm{dL}$ \\
\hline \multicolumn{8}{|l|}{ Sexo } \\
\hline Masculino & 27,4 & 26,5 & 73,4 & 71,2 & 163,5 & 210,9 & 204,2 \\
\hline Femenino & 26,7 & 26,0 & 62,5 & 60,9 & 153,2 & 219,6 & 203,2 \\
\hline Total & 27,0 & 26,2 & 67,4 & 65,5 & 157,8 & 215,7 & 203,7 \\
\hline \multicolumn{8}{|l|}{ Edad } \\
\hline De 20 a 29 años & 27,3 & 26,0 & 65,8 & 62,7 & 155,3 & 190,7 & 183,7 \\
\hline De 30 a 39 años & 27,0 & 26,6 & 64,3 & 63,3 & 154,2 & 214,5 & 196,4 \\
\hline De 40 a 49 años & 27,3 & 26,4 & 67,8 & 65,6 & 157,2 & 222,9 & 205,1 \\
\hline De 50 a 59 años & 26,7 & 25,9 & 69,9 & 67,8 & 161,4 & 218,2 & 209,2 \\
\hline Más de 60 años & 27,0 & 26,3 & 65,3 & 63,6 & 155,3 & 208,3 & 201,2 \\
\hline Total & 27,0 & 26,2 & 67,4 & 65,5 & 157,8 & 215,7 & 203,7 \\
\hline \multicolumn{8}{|l|}{ Función } \\
\hline Administrativo & 27,2 & 26,2 & 65,0 & 62,4 & 154,4 & 207,5 & 202,0 \\
\hline Apoyo Administrativo & 25,7 & 25,0 & 66,9 & 65,2 & 161,1 & 208,4 & 196,7 \\
\hline $\begin{array}{l}\text { Atención Medica y } \\
\text { Hospitalización }\end{array}$ & 27,7 & 27,0 & 67,7 & 66,0 & 156,3 & 220,1 & 208,0 \\
\hline $\begin{array}{l}\text { Apoyo Médico y } \\
\text { Hospitalización }\end{array}$ & 26,7 & 26,0 & 70,6 & 68,8 & 162,1 & 225,0 & 203,0 \\
\hline Total & 27,0 & 26,2 & 67,4 & 65,5 & 157,8 & 215,7 & 203,7 \\
\hline
\end{tabular}

por estudios similares a nivel nacional, en los que también se corroboran mayores prevalencias de obesidad en la población adulta peruana ${ }^{(5)}$.

La consejería estuvo orientada a priorizar una mayor ingesta de: frutas, verduras, granos y frutos secos, reducción de carbohidratos y lípidos, con una adecuada proporción de carnes, acompañada de actividad física durante 10 minutos. De acuerdo a estudios previos, esto desencadena un impacto positivo en el estilo de vida del trabajador y evidencia disminución de parámetros bioquímicos de riesgo ${ }^{(6,7,8)}$.

El estudio mostro que la consejería nutricional basada en el uso de la dieta mediterránea tuvo efecto directo significativo en la disminución del colesterol total. El proceso de consejería fortalece la adopción de prácticas de nutrición y alimentación saludables, y genera espacios de articulación entre las familias y la organización.

\section{REFERENCIAS BIBLIOGRÁFICAS}

1. Fagerhøi MG, Rollefstad S, Olsen SU, Semb AG. The effect of brief versus individually tailored dietary advice on change in diet, lipids and blood pressure in patients with inflammatory joint disease. Food Nutr Res. 2018;62. DOI: 10.29219/fnr.v62.1512

2. Polak R, Dacey M, Phillips EM. Time for food - training physiatrists in nutritional prescription. J Rehabil Med. 2017;49(2):10612. DOI: 10.2340/165019772178

3. Estruch R, Camafort M. Dieta mediterránea y perfil lipidico plasmático. Rev Esp Cardiol. 2015;68(4):279-81. DOI: 10.1016/j.recesp.2014.11.024

4. Sánchez Muñoz-Torrero JF, Crespo L, Ramírez $\mathrm{JM}$, Álvarez F, Luengo J, Mateos J, et al. Efectos de un consejo nutricional simple en pacientes de alto riesgo vascular. Clin Investig Arterioscler. 2011;23(1):8-14. DOI: 10.1016/j.arteri.2010.11.001

5. Pajuelo-Ramírez J. La obesidad en el Perú. Anales de la Facultad de Medicina. 2017;78(2):179-85. DOI: 10.15381/anales.v78i2.13214

6. Parra BE, Manjarrés LM, Velásquez CM, Agudelo GM, Estrada A, Uscátegui RM, et al. Perfil lipídico y consumo de frutas y verduras en un grupo de jóvenes de 10 a 19 años, según el indice de masa corporal. Revista Colombiana de Cardiología. 2015;22(2):72-80. DOI: 10.1016/j.rccar.2014.10.005

7. Pérez-Jiménez F, Pascual V, Meco JF, Pérez Martinez P, Delgado Lista J, Domenech M, et al. Documento de recomendaciones de la SEA 2018. El estilo de vida en la prevención cardiovascular. Clin Investig Arterioscler. 2018;30(6):280-310. DOI: 10.1016/j.arteri.2018.06.005

8. Rubio Pérez FJ, Franco Bonafonte L, Ibarretxe Guerediaga D, Oyon Belaza MP, Ugarte Peyron P. Efecto de un programa de ejercicio físico individualizado sobre el perfil lipídico en pacientes sedentarios con factores de riesgo cardiovascular. Clínica e Investigación en Arteriosclerosis. 2017;29(5):201-8. DOI: 10.1016/j.arteri.2017.02.002 